\title{
Modeling Production Workflows in a Mass Customization Era
}

\author{
Nadine Keddis, Gerd Kainz, Alois Zoitl \\ fortiss $\mathrm{GmbH}$ \\ Guerickestr. 25, 80805 Munich, Germany \\ \{keddis, kainz, zoitl\}@fortiss.org
}

\author{
Alois Knoll \\ Tech. Univ. Muenchen TUM \\ Boltzmannstr. 3, 85748 Garching, Germany \\ knoll@in.tum.de
}

\begin{abstract}
Future manufacturing systems are required to be adaptable and react quickly on changes in markets and demands. Additionally, they should be able to support individualized products that are tailored to customer needs. Flexibility can be increased through decoupling product descriptions from manufacturing systems. Production processes are no longer described based on available resources. Instead, a generic description with all required parameters is defined once and later used with different factory setups. We propose a list of parameters and information that should be included in such a generic product description. Afterwards, we define possible structures for product descriptions that can typically occur in manufacturing systems. We evaluate the approach on an experimental setup used for educational purposes.
\end{abstract}

\section{INTRODUCTION}

Future manufacturing is characterized by a shift towards mass customization [1], [2]. More and more products are personalized. This results in a large number of variants for each product as well as a large number of different products. In the footwear industry, for example, shoes are offered in different sizes, colors, using different fabrics, and with different decoration elements. A mass production is not feasible with such a palette of products and customers would not accept it. This phenomenon is also valid for other industries because of increased customer expectations [3] and saturation of markets [4]. At the same time, the number of different products is increasing and there is a shift towards production with lot size 1 . This leads to a higher complexity in production. In order to cope with the increased complexity and the high number of variants while decreasing the quantity per product, changeable manufacturing systems [5] are necessary.

Current manufacturing systems are designed to fulfill one dedicated task. This leads to optimized and fixed production plans for efficient production of single products. The production plans are not explicitly modeled, but instead are highly integrated with the control software of the manufacturing system. Therefore, changes in products or product requirements typically result in parts of the production systems having to be reconfigured, reprogrammed, or even replaced. Changes always involve manual effort [6] because available IT systems are too inflexible [7], [8]. Current IT systems in automation systems are not agile enough to adapt to changes resulting from flexible customer demands.

In recent years IT is more and more finding its way into the manufacturing domain [9]. Established technologies and concepts from the IT domain are starting to be integrated in the manufacturing domain. Managing business processes through workflow management systems is a development that was possible due to huge improvements in IT [10]. Production processes are in a way similar to business processes. However, they are not yet explicitly modeled. Therefore, not many techniques exist to manage them and automatically adapt to changes based on predefined workflows. In the business and software domains various modeling languages and mechanisms have been developed to describe business processes. Examples are the Business Process Modeling Notation (BPMN), workflow patterns, and petri nets. Additionally, execution languages and tools were developed to automatically implement the processes. Especially service-oriented architectures (SOA) benefit from this development.

This paper investigates how manufacturing processes and workflows can be modeled using concepts adopted from the IT domain. The modeling is based on the analysis of required information for manufacturing workflows with a later automatic execution of workflows in mind. The analysis resulted in a pattern classification of workflows in four main categories.

The remainder of this paper is structured as follows: In Section II used terms are briefly described. Section III gives an overview of available work in the field of workflow modeling. The scope of this evaluation is the application of workflows in the manufacturing domain. The required information for workflow models is explained in Section IV. The different workflow structures of the manufacturing domain are presented in Section V. A possible modeling language and its usage in a simplified industrial example are presented in Section VI. Section VII discusses benefits and problems of this approach. Finally, Section VIII concludes the paper.

\section{TERMinOLOGY}

Before we explain the requirements for modeling workflows for adaptable manufacturing systems, we give a brief overview of the terminology used in this paper:

Process: A process in this context refers to a transformation of or a change to a product that happens during production. It refers to the physical process. However, the description is high-level and does not involve resource-related information. Examples are welding, gluing, transporting or assembly. 
Production Step: A production step denotes the description of a process with all its parameters that occur at one point in time during production. It contains information about the process like its name and its parameters.

Workflow: A workflow combines different production steps into a complete description of the production. It includes the production steps that are involved in production as well as the order in which these steps have to be executed, in order to result in the right product.

Product: A product is a result of several production steps. When talking about a product, we refer to either intermediate products or final products that appear at the end of a workflow.

\section{RELATED WORK}

While there has been much work in improving the adaptability of manufacturing systems, the topic of explicit modeling of production workflows is still in the beginning. There have been approaches to modularize production workflows and standardize the description of processes. The German guideline VDI 2860 [11] is such an example. The goal is to define reoccurring tasks and processes in the assembly domain with their required parameters. These tasks are then classified into different classes and given a standardized symbol for representation. This description is similar to what is required for modeling production workflows. However, the focus of this guideline is on the process itself and not on the product description. Additionally, it does not consider how these different processes can be combined to result in a final product.

The German guideline VDI 3682 [12] defines a formal process description for the process industry. It aims at dividing continuous processes in discrete steps. The description is based on the Polke's phase model [13].

In project management several techniques exist to describe workflows in general. Examples are Project Evaluation and Review Technique (PERT), Critical Path Method (CPM), and Gantt charts [14]. Tasks are described in an abstract way. The timing is more relevant in such approaches than the detailed description of the different tasks. However, tasks dependencies are described well in such approaches, which is also relevant for manufacturing processes. Draht et al. [15] describe how PERT and Gantt charts can be integrated into AutomationML using sequential function charts.

Zhang et al. [16] propose the combination of workflow management with agent-based systems. In their work a workflow management system is used to execute the production process that is divided among several agents. However, this work does not define how such a process plan or workflow is described and what information needs to be modeled in order to execute the production process autonomously. The used example describes the process in a textual form. In addition, the workflow is not decoupled from the production resources that are used in the manufacturing system.

The work of Rehage et al. [17] confirms that production resources as well as processes have to be described in the same manner to support the design of manufacturing systems. However, their focus is on the design and conceptual planning of a manufacturing system. The aim is supporting the user in finding matching resources. Our work is directed at using the process description for automatic execution of the processes in adaptive manufacturing environments. In this case, the planning system uses the process information to directly assign respective resources without human intervention. Nevertheless, some attributes that are used for their process description can be reused in this context as well.

Zor et al. [8] propose using the BPMN language for modeling manufacturing processes. BPMN is mainly used for modeling business processes. In their work BPMN is used to model value streams in production lines, which means they describe the necessary actions to get from raw material to a product. However, they focus on production flows from customer to manufacturing system and neglect the production process itself. Moreover, they identify the gaps in BPMN for describing such manufacturing processes.

In a later work of Zor et al. [18] they propose extensions to BPMN for the manufacturing domain. These extensions enable modeling of the production process while involving the used manufacturing resources. This couples the process to the machines, which is the opposite of what we are trying to achieve for adaptable manufacturing systems. However, they suggest a modeling technique that is useful for documenting processes in the manufacturing domain, but it is not abstract enough for production planning purposes.

\section{MODELING WORKFLOWS}

In order to increase adaptability of manufacturing systems, a decoupling of workflows from the actual manufacturing system is necessary. Currently, the workflow of the production is not modeled explicitly. Rather, it is implicitly included in the control software of each resource in the manufacturing system. Therefore, workflows cannot be reused in other factories and it is hard to change them. Whenever a change is introduced in the workflow, the control software has to be reprogrammed or reconfigured. This is very costly and time-consuming. With a separate representation of the workflow, parts of the control software can be automatically generated or even directly executed. To achieve this, the workflow model has to fulfill certain requirements that will be discussed in detail in this section. The workflows are described with formal modeling techniques that can be represented in machine-readable format.

\section{A. Modular Production Steps}

The workflow within a manufacturing system should describe each step of the production. In the first step the production process has to be modularized. Each process used to produce a product should be encapsulated into a single step that can be used within a workflow. The steps should abstract from specific implementations and technical information related to the factory setup. Instead, a step should describe the process in terms of what has to be done to transform the raw material into the next product. Examples for steps within a workflow are cutting, gluing, or sewing. Ideally, a workflow would be composed of standardized production steps only. The 
German guideline VDI 2860 [11] tries to classify different manufacturing processes. However, standardization effort is necessary to establish production steps for manufacturing.

\section{B. Production-Relevant Data}

Additional information is required to be able to directly control the resources using the workflow description and add product specific parameters. Required information can be added to production steps using attributes for each step. In the following, such attributes are described in more detail.

Raw Material and Products: Since a workflow can involve several different raw materials, describing the materials that are used within a production step is necessary. Examples for different materials are metal, plastic, or different fabrics. Depending on the material, different tools may be necessary to perform a production step. As an example, drilling plastic and drilling metal might require a different drill. However, both steps are described as a drilling step with different attributes. Beside the type of material, other material parameters can be specified in the production step. Color attributes, for example, could be included to define customer requirements. Another aspect that needs to be considered is the physical input and output of productions steps. This is either a raw material or a product. Similarly to raw materials, products have to be described as well. For production steps that do not change the material or product (e.g., transportation), the same input description can be used for the output. For production steps that transform the material or product (e.g., gluing), the description of the output has to be updated. During the gluing process, two materials are glued together to result in a new product. All these different attributes can be specified explicitly or combined in one identifier. In the latter case, a database with a mapping from identifier to attributes is necessary.

Geometry: Another attribute is the geometry of the material or product. Similar to the used material, different resources might be necessary to perform the same production steps but for products with different geometries. Depending on the geometry different grippers or palettes might be necessary. The geometry also includes the measurements of the materials and the products to be able to infer whether for example available conveyors can be used to transport the products from one resource to another.

Process-Related Data: Depending on the process, different additional parameters might be necessary. One example is the cutting line that is required for a specific product in a cutting step. The same applies to the sewing or gluing line in a sewing or gluing process. Similarly, it is necessary to specify the exact position where the material should be placed in a pick\&place process. Some processes might require a specific timing in order to result in the right product. For example it is necessary to know how long a material has to be heated in order to achieve certain characteristics.

Error Tolerance and Quality Requirements: For some processes an error tolerance can be specified to distinguish between good and faulty products. For a cutting process the error tolerance would specify the percentage that a cut material

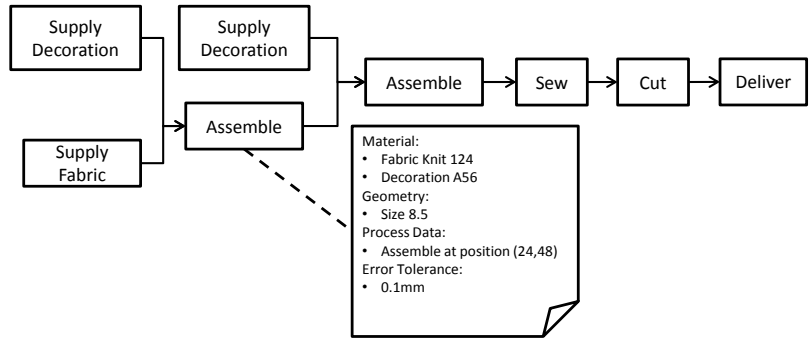

Fig. 1. Production plan for a shoe. Boxes represent the required capabilities and arrows the dependencies between them.

can differ from the specification. This percentage defines how much bigger or smaller the material can be after cutting compared to the given template. The quality requirements have the same goal as the error tolerance. They define quality measures that must be fulfilled in this step.

\section{Combining Production Steps to Workflows}

In addition to defining modular production steps for a specific product and including all relevant parameters, dependencies between required production steps have to be described. Dependencies determine the order of execution within the workflow. Each step in the workflow model represents the physical process that can be performed by a resource within the manufacturing system. The order is important for scheduling decisions. Dependencies can be described by defining either the predecessor or the successor for each step. Each step can have zero, one, or more predecessors or successors respectively. Dependencies are transitive, so they need to be specified only for the immediate predecessor or successor. For the moment the approach is limited to acyclic production plans, but cyclic dependencies are planned in the future.

A simplified example is illustrated in Figure 1. The example shows the necessary steps to produce a shoe. Steps with no incoming arrows represent starting points for the production and are independent of other steps. Steps with no outgoing arrows represent the final production steps. Several final steps can exist if the workflow describes a production that results in more than one product.

\section{WORKFLOW STRUCTURES}

Based on the analysis of manufacturing systems and looking at discrete and process manufacturing [19], manufacturing workflows can be described using four basic structures ${ }^{1}$. The basic structures are sequential, synchronization, fork, and selective as depicted in Figure 2. The notation used here is just for explanation purposes and is not intended as the modeling notation. Every manufacturing process can be described using either one of these structures or a combination of them. Additionally, the analysis resulted in two more complex structures that are an extension of the basic ones. In the following, the structures are described in more detail. We describe the patterns for intermediate production steps since we can extend

\footnotetext{
${ }^{1}$ For simplification, this paper discusses only specification of workflows without errors. However, the suggested approach also allows the specification of actions that should be performed in case of an error.
} 


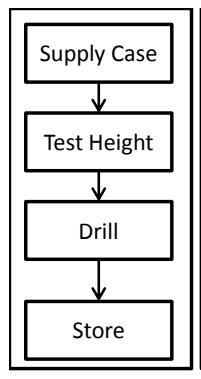

(a)

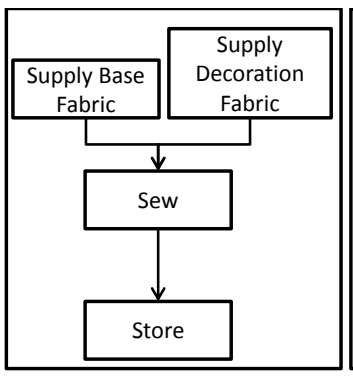

(b)

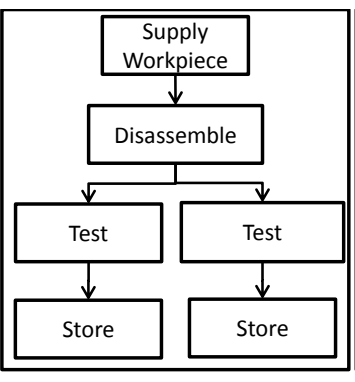

(c)

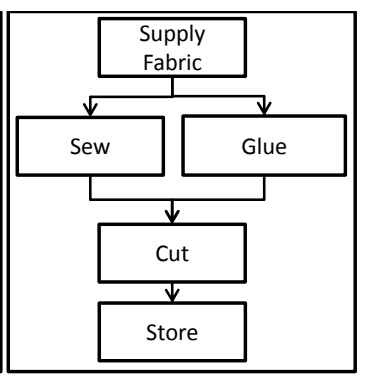

(d)

Fig. 2. Different workflow structures: (a) A sequential, (b) a synchronization, (c) a fork, (d) and a selective workflow.

each workflow with a dummy start and end node. The start node starts the workflow upon request and triggers the first steps in a workflow. The end node synchronizes the end steps of a workflow.

\section{A. Sequential Workflows}

A sequential step describes a production step that can be started after the preceding production step has finished its execution. All production steps in an assembly line are sequential activities, because the next step cannot start before the previous one has finished. Therefore, all consecutive steps in a production process can be described using such a structure. An example is illustrated in Figure 2a.

\section{B. Synchronization Workflows}

A synchronization step describes a production step that can be started after multiple parallel production steps have finished. The activity synchronizes two or more different parallel production steps as shown in Figure $2 \mathrm{~b}$ in the Sew step. The synchronization step can only be started after all parallel production steps that precede it finish their execution. In manufacturing, parallel steps are mainly possible when the production steps are performed on different materials or products. However, exceptions like cooling a product while transporting it are possible. Typically, the different materials and products are combined together in the synchronization step which results in the next product. Therefore, after the synchronization step the several incoming production threads are combined in one active production thread.

\section{Fork Workflows}

A fork workflow is the counterpart to a synchronization workflow. In a fork workflow there is one activity that splits the production in two parallel processes. When this activity finishes two parallel active production threads are started. Typically such an activity involves splitting one part into several ones and performing different actions on the different parts and materials.

\section{Selection Workflows}

A selection workflow is used when multiple production processes are possible to achieve a production step. The different production processes must result in the same product; otherwise they cannot be used interchangeably. A selection workflow is always nested between two production steps. In the example in Figure 2c the steps Sew and Glue represent the selection workflow. They are nested between the steps Supply Fabric and Cut. The production step after the selection workflow can be started when one of the previous production steps has finished. During a production only one of the branches in a selection is active. The others are not used to produce the final product. It is an exclusive OR relationship. In the example of Figure 2c this means that either Sew or Glue is going to be used in the production.

\section{E. Pairing Workflows}

In pairing workflows, two or more parallel workflows have to be synchronized because they belong to the same product. However, the parallel production processes are independent of each other. A pairing workflow can consist of two or more sequential, synchronization, fork, or selection workflows, or a combination of them. Figure 3a shows an example of a pairing structure consisting of two sequential workflows. Pairing workflows can be transformed into synchronization workflows by adding a synchronization step at the end of the parallel workflows. This step is usually the packaging step.

\section{F. Arbitrary Order}

For production steps that are independent, it should be possible to specify that they can be performed in an arbitrary order. This also applies to some quality assurance steps that can take place during the process. When several tests have to be done in the same step, it is possible to specify that the order is arbitrary. As a simplified example, let us look at a drilling process with an additional requirement regarding the height of the product. The process involves the supply of the object to be drilled. Before the drilling, two tests have to be taken to ensure that the step will be performed correctly. The first test checks the height of the object to make sure that it fulfills the requirement. The second ensures that the object has the right orientation so that the drilling process is successful. For the end-result, the order in which these two tests are performed is irrelevant. It is only important that they are both performed. Figure 3 illustrates this example.

When there is a sequence of production steps that can be done in an arbitrary order, it should be possible to decide at run-time which order is more suitable. This depends on the factory setup, the machine availability, and the scheduling 
TABLE I

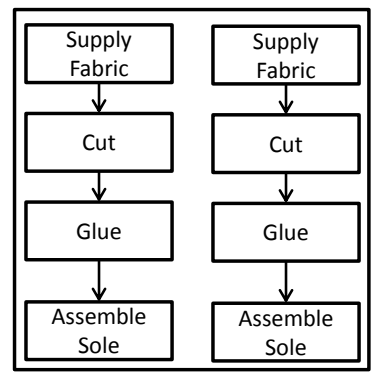

(a)

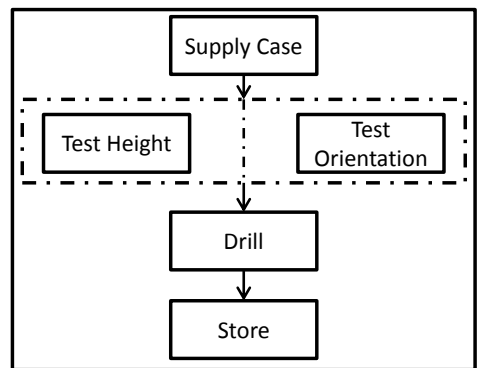

(b)
Fig. 3. Complex workflows: (a) Production of left and right shoe, (b) arbitrary steps are modeled in dashed-line steps.

criteria. In some cases, especially with quality tests, a certain order is more efficient regardless of factory setup or machine availability. This is the case for the height test in the simplified example. Since the height test determines whether the object will be used or dismissed, it is more efficient to have this test earlier in the workflow. This will avoid unnecessary production steps and therefore reduce cost and time.

\section{BRINGING THE WORKFlOW MOdel TO LIFE}

The goal is to use workflows in an automated planning and scheduling system to determine the production schedule. The workflow is modeled by a process expert, who has knowledge about the processes and the different production steps. After modeling the workflow for a product, the planning and scheduling system can use the modeled information to decide which resources should be used in the different production steps and in which order. Ideally, this should be done without user intervention and through considering the available resources and their relations. To evaluate whether modeling workflows can improve the flexibility and enable automated schedule generation, we modeled products for a simplified manufacturing system used for educational purposes.

\section{A. Experimental Setup}

The setup consists of different resources from the Festo Modular Production Systems that can be combined together to form a manufacturing system. One possible setup is shown in Figure 4. The setup supports different products: thermometers, hygrometers, boxes, and workpieces in three different colors and with different characteristics. For each of these products a workflow has to be modeled. Different production steps can be included in the workflow depending on the desired product characteristics. Examples are listed in Table I. The user can set-up the factory using the stations he wants to include. These stations log onto a planning and scheduling system autonomously as described in previous work [20]. Afterwards, the user can choose the different products that should be produced and the system automatically generates a feasible schedule and controls the production.

\section{B. Modeling Workflows}

The different products that we have in our setup are modeled using the Eclipse Modeling Framework (EMF) [21]. EMF
PRODUCTION STEPS THAT ARE USED IN THE EXPERIMENTAL SETUP.

\begin{tabular}{|c|l|}
\hline Production Step & Description \\
\hline Supply & $\begin{array}{l}\text { Supply material such as cases, boxes, sensors, } \\
\text { covers, etc. } \\
\text { Different tests (e.g., Test Height } \text { and Test } \\
\text { Orientation). } \\
\text { Drilling a whole in the workpiece. } \\
\text { Putting two materials together (e.g., a pick \& } \\
\text { place operation). }\end{array}$ \\
Assemble & $\begin{array}{l}\text { Buffering a number of workpieces or products. } \\
\text { Sorting workpieces or products according to a } \\
\text { sorting criterion. }\end{array}$ \\
Store &
\end{tabular}

is a key reference in the world of model-driven software development. It offers a framework for modeling applications and generating classes for the different elements in the models.

In EMF a metamodel is defined to describe how valid workflow models should be built. The metamodel for workflows is illustrated in Figure 5. Each workflow contains a number of production steps as well as the dependencies between them. Each production step describes which processes are required in this step as well as the required parameters as described earlier. Additionally, the production step contains a reference to products or materials that are used in this step. To model the details of the workflow, two additional models are required: A material/product model and a process model. The material/product model defines all the materials and products that are possible within the setup. The process model defines a set of processes that can be used to describe the required production steps as well as the processes of the different production resources. For each product that can be produced by the experimental setup, an instance of a workflow was modeled in EMF. The models is stored in an XML-like file (.xmi). In this way, we could make use of the models in our $\mathrm{C}++$ application for planning and scheduling production while considering our available factory setup.

\section{DISCUSSION}

Modeling the workflows independently from the factory setup allows changing the factory setup without having to adapt the planning software. The planning software automatically uses the workflow models to map production steps to

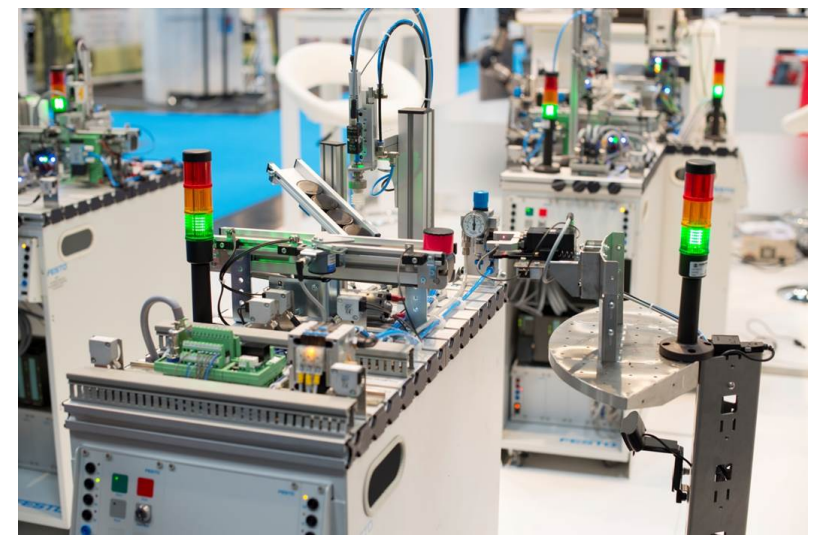

Fig. 4. One possible setup of the manufacturing system used for evaluating the approach. 


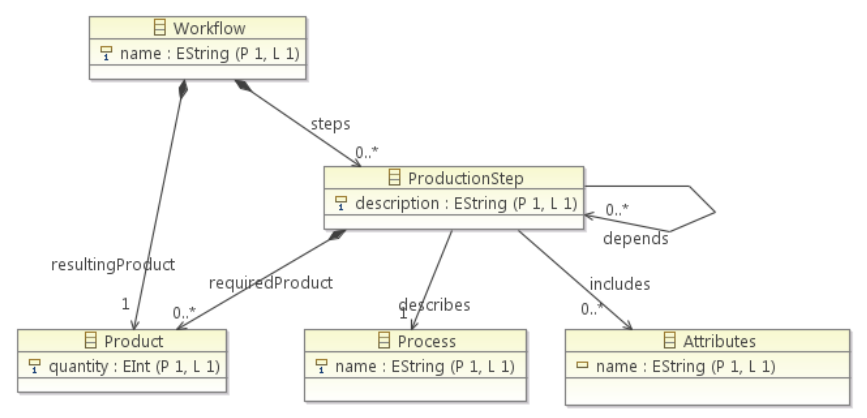

Fig. 5. Metamodel of the workflows

resources and feed the resources with the right parameters that are necessary for a specific product. Using the workflow models, we are able to support different products and arbitrary factory setups. The modeling language used in this paper is a generic modeling language that can be used for different domains and purposes. Since the workflows are described using domain-specific terminology, it is easy for domain experts to model the workflows with their process knowledge. The examples used in the experimental setup show how such explicit workflow models can increase flexibility and decouple the factory from the product.

Languages such as BPMN already include the process notion. Nevertheless, they are not tailored for the manufacturing industry and do not include enough information to automatically control the production yet. Further extensions might be a possibility to make them more suitable for manufacturing. A survey of available languages and their suitability should therefore be conducted. Important aspects that should be considered are the possibility to express domain related parameters as well as the usability for domain experts. Additionally, we need to investigate whether the mentioned information categories are sufficient to cover most cases in the manufacturing domain.

\section{CONCLUSION}

The focus of this paper was to present a general concept for describing workflows in the manufacturing domain. This is motivated by the need to decouple product descriptions from production resources in order to be viable in times of fast paced changes in the production industry. In this paper we explained the information that should be included in such workflow models and gave an overview of the different workflow structures that can occur within a manufacturing system. The approach was then evaluated on an experimental setup used for educational purposes. The evaluation showed that the approach requires the modeling of a workflow once and allows reusing this model for different factory setups. It also allowed the automatic generation of valid schedules and their execution afterwards.

The next step of this work involves evaluating different description languages for modeling such workflows in order to find a suitable mechanism. Additionally, we intend to evaluate whether the stated information is sufficient for larger production systems or whether we need an extension of the attributes of the production steps. Moreover, we plan to look at modeling error handling in workflows as well. The error handling will probably increase the workflow structure categories.

\section{AKNOWLEDGMENT}

This approach is a result of the research and development project "SpeedFactory", which is funded by the German Federal Ministry for Economic Affairs and Energy as part of the technology programme "Autonomics for industry 4.0" and supervised by the German Aerospace Center (DLR) under Grant No. 01MA13002B.

\section{REFERENCES}

[1] S. Hu, X. Zhu, H. Wang, and Y. Koren, "Product Variety and Manufacturing Complexity in Assembly Systems and Supply Chains," CIRP Annals-Manufacturing Technology, vol. 57, no. 1, pp. 45-48, 2008.

[2] Iacocca Institute, "21. Century Manufacturing Enterprise Strategy: An Industry-Led View," Iacocca Institute, Bethlehem, Pennsylvania, Tech. Rep., 1991.

[3] U. Lindemann and G. Baumberger, Individualisierte Produkte. Springer, 2006.

[4] E. Westkämper and M. Decker, Einführung in die Organisation der Produktion. Springer, 2006.

[5] H.-P. Wiendahl, H. ElMaraghy, P. Nyhuis, M.-F. Zäh, H.-H. Wiendahl, N. Duffie, and M. Brieke, "Changeable Manufacturing - Classification, Design and Operation," CIRP Annals-Manufacturing Technology, vol. 56, no. 2, pp. 783-809, 2007.

[6] M. Zäh, G. Reinhart, M. Ostgathe, F. Geiger, and C. Lau, "A Holistic Approach for the Cognitive Control of Production Systems," Advanced Engineering Informatics, vol. 24, no. 3, pp. 300-307, 2010.

[7] O. Sauer and J. Jasperneite, "Adaptive Information Technology in Manufacturing," in CIRP Conference on Manufacturing Systems, Madison, WI, USA, Jun 2011.

[8] S. Zor, K. Görlach, and F. Leymann, "Using BPMN for Modeling Manufacturing Processes," in Proceedings of 43rd CIRP International Conference on Manufacturing Systems, 2010, pp. 515-522.

[9] B. Vogel-Heuser, G. Kegel, K. Bender, and K. Wucherer, "Global information architecture for industrial automation."

[10] W. Van Der Aalst and K. M. Van Hee, Workflow Management: Models, Methods, and Systems. MIT press, 2004.

[11] VDI-Richtlinie 2860, "Montage- und Handhabungstechnik; Handhabungsfunktionen, Handhabungseinrichtungen; Begriffe, Definitionen, Symbole." 1990.

[12] VDI-Richtlinie 3682, "Formalisierte Prozessbeschreibungen," 2005.

[13] M. Polke, "Prozeßleittechnik," 1994.

[14] K. Pearlson and C. S. Saunders, Managing and Using Information Systems: A Strategic Approach. Wiley Hoboken, NJ, 2004.

[15] R. Drath, A. Lüder, J. Peschke, and L. Hundt, "AutomationML - the glue for seamless Automation Engineering," in Emerging Technologies and Factory Automation, 2008. ETFA 2008. IEEE International Conference on. IEEE, 2008, pp. 616-623.

[16] Y. Zhang, G. Q. Huang, T. Qu, and O. Ho, "Agent-based Workflow Management for RFID-enabled Real-Time Reconfigurable Manufacturing," International Journal of Computer Integrated Manufacturing, vol. 23, no. 2, pp. 101-112, 2010.

[17] G. Rehage, F. Bauer, and J. Gausemeier, "Specification Technique for the Consistent Description of Manufacturing Operations and Resources," in Enabling Manufacturing Competitiveness and Economic Sustainability. Springer, 2014, pp. 47-53.

[18] S. Zor, D. Schumm, and F. Leymann, "A Proposal of BPMN Extensions for the Manufacturing Domain," in Proceedings of 44th CIRP International Conference on Manufacturing Systems, 2011.

[19] H. Mersch, D. Behnen, D. Schmitz, U. Epple, C. Brecher, and M. Jarke, "Gemeinsamkeiten und Unterschiede der Prozess-und Fertigungstechnik," at-Automatisierungstechnik Methoden und Anwendungen der Steuerungs-, Regelungs-und Informationstechnik, vol. 59, no. 1, pp. 7-17, 2011.

[20] N. Keddis, G. Kainz, C. Buckl, and A. Knoll, "Towards Adaptable Manufacturing Systems," in IEEE International Conference on Industrial Technology (ICIT), 2013. IEEE, 2013, pp. 1410-1415.

[21] D. Steinberg, F. Budinsky, E. Merks, and M. Paternostro, EMF: Eclipse Modeling Framework. Pearson Education, 2008. 